Abstract

\title{
Representation and Information in Molecular Modelling ${ }^{\dagger}$
}

\author{
Raffaello Potestio \\ Physics Department, University of Trento, Povo, Italy \\ + Presented at the Entropy 2021: The Scientific Tool of the 21st Century, 5-7 May 2021; Available online: \\ https://sciforum.net/conference/Entropy2021/.
}

Published: 5 May 2021

The computational study of soft matter systems lies at the nexus among several disciplines, including material science, biophysics, statistical mechanics, and information theory. Each of them contributes objects of investigation as well as tools and perspectives unique from each field, all of these being necessary to attain a multifaceted picture of complex systems. The construction of in silico models of biomolecules, in particular, requires one to combine the background biological knowledge of a system with the quantitative description of the latter in those terms characteristic of mechanics and statistical physics. This is particularly true in the field of coarse-grained modelling, in which the effort to attain an ever larger accuracy of a model is replaced by the attempt to simplify it as much as possible, while at the same time retaining those essential features which make the model predictive. This talk will provide an overview of the problem of designing effective, coarse-grained models of large biomolecules. Particular attention will be posed on the issue of representability and mapping, the preservation of information content, and the extraction of biological knowledge from resolution modulation.

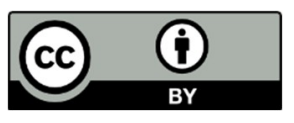

(C) 2021 by the author. Licensee MDPI, Basel, Switzerland. This article is an open access article distributed under the terms and conditions of the Creative Commons Attribution (CC BY) license (http://creativecommons.org/licenses/by/4.0/). 